\title{
METAL COMPOUNDS OF THE TCNX REDOX SYSTEMS - FROM ELECTRON TRANSFER TO SPIN DISTRIBUTION AND MAGNETISM
}

\author{
WOLFGANG KAIM
}

Institut fuer Anorganische Chemie, Universitaet Stuttgart, Pfaffenwaldring 55, D-70550 Stuttgart, Germany

Dedicated to the memory of Jaime Retuert, Dr.rer.nat. graduate from the Universitaet Stuttgart in 1972

\begin{abstract}
The TCNX ligands, TCNE (tetracyanoethene), TCNQ (7,7,8,8-tetracyano- $p$-quinodimethane), and TCNB (1,2,4,5-tetracyanobenzene), are capable of bridging up to four metal centers in discrete molecular complexes or coordination polymers. In addition, the highly non-innocent TCNX ligands may exist in neutral $\pi$ acceptor form, as stable monoanionic radicals, or as dianions, which has generated a unique coordination chemistry. Several such compounds, particular those involving the TCNX- bridges, have found interest for molecular magnetism studies, organic conductor research, and in concepts of molecular computing. This contribution focuses on the chemical potential for new materials and on their physical characterization, especially with respect to the TCNX/metal electron transfer and to the spin distribution in paramagnetic states.
\end{abstract}

Keywords: Electron transfer, Non-innocent ligands, Spin distribution, TCNQ, Tetracyanoethene

The ability of the $\pi$ conjugated tetranitrile ligands TCNE, TCNQ and TCNB (Scheme 1) to bridge up to four metal centers (Scheme 2) and to exist in the neutral $\pi$ acceptor form, as stable monoanionic radicals, or as dianions (Scheme 3) has generated a unique coordination chemistry (Scheme 2) ${ }^{1}$ which has found potential applications in molecular magnetism ${ }^{2,3}$, organic conductor research ${ }^{4}$, and in concepts for molecular computing ${ }^{5}$.

Scheme 1

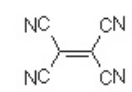

tetracyanoethene (TCNE)

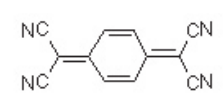

$7,7,8,8$-tetracyano- $\mathrm{p}$-quinodimethane (TCNQ)

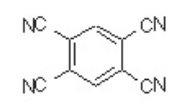

$1,2,4,5$-tetracyanobenzene (TCNB)

\section{Scheme 2}

$$
\begin{aligned}
& \left(\eta^{2}-\mathrm{TCNX}\right)\left(\mathrm{ML}_{n}\right) \quad \pi \text { complex, side-on, } \mathrm{C} \underset{\frac{\mathrm{M}}{\mathrm{M}}}{\mathrm{C}} \\
& \mathrm{TCNX}+\mathrm{ML}_{\mathrm{n}} \\
& \downarrow \\
& \left(\eta^{1}-\mathrm{TCNX}\right)(\mathrm{ML}) \quad \sigma \text { complex, end-on, } \quad-\mathrm{C} \equiv \mathrm{N} \cdots \mathrm{M} \\
& \downarrow+\mathrm{ML}_{\mathrm{n}} \\
& {\left[\left(\mu, \eta^{1}: \eta^{1}-\mathrm{TCNX}\right)\left(\mathrm{ML}_{\mathrm{r}}\right)_{2}\right]_{\mathrm{x}}} \\
& \downarrow+\mathrm{ML}_{\mathrm{n}} \\
& \left(\mu_{3}, \eta^{1}: \eta^{1}: \eta^{1}-\mathrm{TCNX}\right)\left(\mathrm{ML}_{\mathrm{N}}\right)_{3} \\
& \downarrow+\mathrm{ML}_{\mathrm{n}} \\
& \left(\mu_{4}, \eta^{1}: \eta^{1}: \eta^{1}: \eta^{1}-\mathrm{TCNX}\right)\left(\mathrm{ML}_{\mathrm{N}}\right)_{4}
\end{aligned}
$$

\section{Scheme 3}

$$
\left(\mathrm{TCNX}^{\circ}\right)\left(\mathrm{M}^{\mathrm{k}} \mathrm{L}_{\mathrm{n}}\right) \longleftrightarrow\left(\mathrm{TCNX}^{*}\right)\left(\mathrm{M}^{\mathrm{k}+1} \mathrm{~L}_{\mathrm{n}}\right) \longleftrightarrow\left(\mathrm{TCNX}^{2-}\right)\left(\mathrm{M}^{\mathrm{k}+2} \mathrm{~L}_{\mathrm{n}}\right)
$$

Whereas only mono- and dinuclear discrete compounds could be obtained with organometallic complex fragments of $\mathrm{Ti}^{6}, \mathrm{~V}^{7}, \mathrm{Cr}^{8}$ and $\mathrm{Co}^{7}$ it has been possible to establish the full coordinative saturation in tetranuclear complexes of manganese (TCNE, TCNQ) ${ }^{8,9}$ rhenium (TCNQ) ${ }^{10}$ ruthenium (TCNE, TCNQ, TCNB) ${ }^{11}$ osmium (TCNE, TCNQ, TCNB) ${ }^{12}$ and copper (TCNE, TCNQ, TCNB) ${ }^{13}$. For iron there has been a report by Diaz and Arancibia describing the complex $\left\{\left(\mu_{4}-\mathrm{TCNQ}\right)\left[\mathrm{Fe}(\mathrm{dppe})\left(\mathrm{C}_{5} \mathrm{H}_{5}\right)\right]_{4}\right\}^{4+}$, dppe $=1,2$-bis(diphenylphosph ino)ethane ${ }^{14}$ while we could recently describe $\left\{\left(\mu_{4}-\mathrm{TCNE}\right)\left[\mathrm{Fe}(\mathrm{CO})_{2}\left(\mathrm{C}_{5} \mathrm{H}_{5}\right)\right]_{4}\right\}^{4+}$ 15. Except for the latter and for $\left\{\left(\mu_{4}-\mathrm{TCNQ}\right)\left[\operatorname{Re}(\mathrm{CO})_{3}(\mathrm{bpy})\right]_{4}\right\}^{\}^{+}}$all these compounds showed strong evidence from spectroscopy and electrochemistry for considerable metal-to-ligand electron transfer $\delta$ in the ground state of $\left(\mu^{4}-\right.$ $\left.\mathrm{TCNX}^{\delta-}\right)\left(\mathrm{M}^{\mathrm{k}+\delta / 4} \mathrm{~L}_{\mathrm{n}}\right)_{4}($ Scheme 4).

\section{Scheme 4} $\left.{ }^{4} \mathrm{~L}_{\mathrm{n}}\right)_{4}$

$\left(\mu_{4}-\mathrm{TCNX}^{\circ}\right)\left(\mathrm{M}^{\mathrm{k}} \mathrm{L}_{\mathrm{n}}\right)_{4} \longleftrightarrow\left(\mu_{4}-\mathrm{TCNX}^{2-}\right)\left(\mathrm{M}^{\mathrm{k}+0.5} \mathrm{~L}_{\mathrm{n}}\right)_{4}: \quad\left(\mu_{4}-\mathrm{TCNX}^{\delta}\right)\left(\mathrm{M}^{\mathrm{k}+\delta /}\right.$

In addition to the discrete tetranuclear complexes mentioned above, several coordination polymers were reported with $\mu_{4}$-TCNE ${ }^{16}$ or $\mu_{4}$-TCNQ ${ }^{17}$ of which some exhibit structural evidence for intramolecular metal-to-ligand electron transfer.

Such coordination polymers have found particular attention because the first room-temperature molecule-based magnet, $\mathrm{V}^{\mathrm{II}}\left(\mathrm{TCNE}^{*}\right)^{\bullet} \mathrm{x}$ solvent, is assumed to consist of a three-dimensional coordination polymeric network involving the bridging TCNE anion radical 2a,18. Unfortunately, the absence of detailed structural information beyond XANES/EXAFS data ${ }^{19}$ precluded a better understanding of this fascinating amorphous material. Infrared spectroscopy has also been extensively used in analyzing the coordination mode and/or oxidation state of TCNE ${ }^{1}$ in non-crystalline materials. It should be added that another (TCNE*-)-containing compound, $\left[\mathrm{Fe}\left(\mathrm{C}_{5} \mathrm{Me}_{5}\right)\right](\mathrm{TCNE})$, had been presented by Miller et al. as a ferromagnetic material $\left(T_{c}=4.2 \mathrm{~K}\right)$ ${ }^{20}$, however, the anion radical and the decamethylferrocenium cation are not directly coordinated in this ion-pair compound.

The importance to establish and identify "physical oxidation states" ${ }^{21}$ in complexes involving non-innocent ${ }^{10 b}$ ligands is thus obvious, especially when dealing with oligonuclear compounds of redox-active molecular bridges. Using group 8 metals we have focussed on tetranuclear complexes of TCNX (TCNE, TCNQ, TCNB) with two classical, widely used $\mathrm{d}^{6}$ metal complex fragments, the readily oxidizable $\left[\mathrm{Ru}\left(\mathrm{NH}_{3}\right)_{5}\right]^{2+}$ (Scheme 5, cf. the mixed-valent CreutzTaube ion $\left.{ }^{22}\right)$, and the organometallic prototype $\left[\mathrm{Fe}(\mathrm{CO})_{2}\left(\mathrm{C}_{5} \mathrm{H}_{5}\right)\right]^{+}$(Scheme 6).

Scheme 5

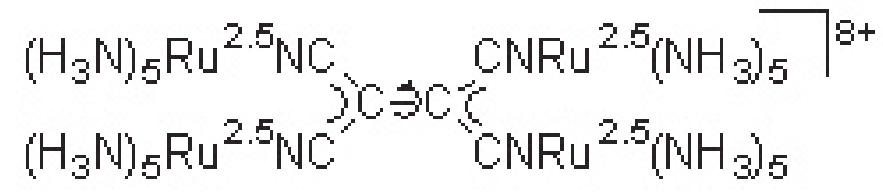

Experimental evidence including electrochemical potentials, magnetic behavior $11 \mathrm{~b}$, charge transfer absorption spectra and infrared vibrational spectroscopy has suggested negligible metal-to-ligand electron transfer in the ground states of $\left\{\left(\mu_{4}-\mathrm{TCNX}^{\circ}\right)\left[\mathrm{Fe}^{\mathrm{II}}(\mathrm{CO})_{2}\left(\mathrm{C}_{5} \mathrm{H}_{5}\right)\right]_{4}\right\}^{4+15}$ whereas extensive such interaction according to formulation $\left\{\left(\mu_{4}-\mathrm{TCNX}^{2-}\right)\left[\mathrm{Ru}^{2.5}\left(\mathrm{NH}_{3}\right)_{5}\right]_{4}\right\}^{8+}$ was proposed for the pentaammineruthenium systems with TCNE and TCNQ 1la,b. In the absence of experimental structures e.g. from single crystal X-ray diffraction, DFT methodology was recently applied to confirm and clarify the situation in such discrete model complexes for coordination polymeric 
materials ${ }^{11 c, 15 b}$. The size, structural alternatives and the complicated electronic structures required the use of advanced DFT procedures to assess the electronic situation with sufficient confidence.

Scheme 6

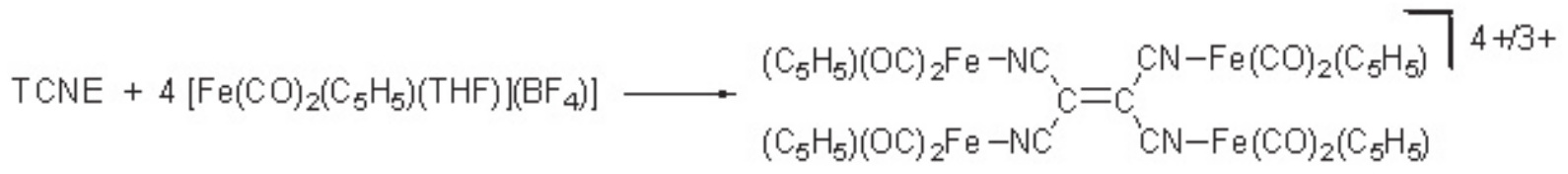

As with the crystallographically confirmed tetrarhenium complex $\left\{\left(\mu_{-}-\right.\right.$ TCNQ $\left.)\left[\operatorname{Re}(\mathrm{bpy})(\mathrm{CO})_{3}\right]_{4}\right\}^{4+}$ the similar tetrairon species $\left\{\left(\mu_{4}-\mathrm{TCNX}\right)[\mathrm{Fe}(\mathrm{C}\right.$ $\left.\left.\mathrm{O})_{2}\left(\mathrm{C}_{5} \mathrm{H}_{5}\right)\right]_{4}\right\}^{4+}, \mathrm{TCNX}=\mathrm{TCNE}, \mathrm{TCNQ}$, were calculated with TCNX ligand parameters which suggest only small metal-to-ligand electron transfer in the ground state. Most conspicuously, the TCNX $\pi$ ligands remain virtually coplanar, and the bond distances correspond to the bond orders as indicated in Scheme $1^{15 b}$. Slight differences in relation to the free ligands arise from multiple metal coordination. In contrast, significantly reduced TCNE and TCNQ exhibit a twisted framework with considerably lengthened CC double bonds ${ }^{1,10}$. Effects of multiple metal coordination can be studied separately for the complexes of much less $\pi$ accepting TCNB ${ }^{2 c, 11 a}$ which is more difficult to reduce (by more than $1 \mathrm{~V}$ ) than TCNE or TCNB and which is unable to twist into a non-planar state ${ }^{11 a}$.

DFT calculations of the complexes $\left\{\left(\mu_{4}-\mathrm{TCNX}\right)\left[\mathrm{Ru}\left(\mathrm{NH}_{3}\right)_{5}\right]_{4}\right\}^{8+}, \mathrm{TCNX}=$ TCNE, TCNQ, yield very different results ${ }^{11 c}$ : The lowest energy structures correspond to triplet states with considerably twisted moieties RNCCCNRu and $\mathrm{C}_{6} \mathrm{H}_{4}$ (TCNQ). The twist angle is close to $90^{\circ}$ with TCNX $=\mathrm{TCNE}$ and $2 \times$ $39.8^{\circ}$ for the complex with TCNQ. Accordingly, the original CC double bonds are lengthened to $1.544 \AA$ (TCNE complex) and $1.508 \AA$ (TCNQ complex, exocyclic CC). The compounds are thus best described by the limiting formulation $\left(\mathrm{TCNX}^{2}\right)\left(\mathrm{Ru}^{2.5}\right)_{4}$, containing strongly coupled mixed-valent entities $\left[\left(\mathrm{H}_{3} \mathrm{~N}\right)_{5} \mathrm{Ru}-\mathrm{NCCCN}-\mathrm{Ru}\left(\mathrm{NH}_{3}\right)_{5}\right]^{4+}{ }^{43}$ which do not interact strongly in a ferromagnetic-type interaction ${ }^{11 \mathrm{~b}}$

Using DFT calculations for the remarkable tetraruthenium complex ions $\left\{\left(\mu_{4}-\mathrm{TCNX}\right)\left[\mathrm{Ru}\left(\mathrm{NH}_{3}\right)_{5}\right]_{4}\right\}^{8+}, \mathrm{TCNX}=\mathrm{TCNE}$ and TCNQ, we can thus provide consistent computational evidence for the model 11a,b invoking weakly interacting pairs of strongly ( $\mathrm{NCCCN}-$ )-coupled $\left(\mathrm{Ru}^{2.5}\right)_{2}$ containing dimers. The near orthogonality in the triplet ground state configurations and the computed $\mathrm{C}-\mathrm{C}$ bond distances point to extensive intramolecular metalto-ligand electron transfer; in contrast to the $\left(\mathrm{TCNQ}^{\circ}\right)\left(\mathrm{Re}^{\mathrm{r}}\right)_{4}$ situation of $\left\{\left(\mu_{4}\right.\right.$ TCNQ) $\left.\left[\operatorname{Re}(\text { bpy })(\mathrm{CO})_{3}\right]_{4}\right\}^{4+}$ or the $\left(\mathrm{TCNX}^{\circ}\right)\left(\mathrm{Fe}^{\circ}\right)_{4}$ configuration of $\left\{\left(\mu_{4}-\mathrm{TCN}\right.\right.$ $\left.\mathrm{X})\left[\mathrm{Fe}(\mathrm{CO})_{2}\left(\mathrm{C}_{5} \mathrm{H}_{5}\right)\right]_{4}\right\}^{4+}$, the $\left\{\left(\mu_{4}-\mathrm{TCNX}\right)\left[\mathrm{Ru}\left(\mathrm{NH}_{3}\right)_{5}\right]_{4}\right\}^{8+}$ ions thus represent unconventional mixed-valent species $\left(\mathrm{TCNX}^{2-}\right)\left(\mathrm{Ru}^{2-5}\right)_{4}{ }^{24}$. Whereas the twoelectron oxidation of $\left\{\left(\mu_{4}-\mathrm{TCNX}\right)\left[\mathrm{Ru}\left(\mathrm{NH}_{3}\right)_{5}\right]_{4}\right\}^{8+}$ to $\left(\mathrm{TCNX}^{2-}\right)\left(\mathrm{Ru}^{\mathrm{III}}\right)_{4}$ is in agreement with the presence of two weakly coupled redox-active molecular entities RuNCCCNRu, the one-electron reduction of the complex involving ligand oxidation to a radical species $\left(\mu_{4}-\mathrm{TCNX}^{*}\right)\left(\mathrm{Ru}^{\mathrm{II}}\right)_{4}$ before ending in $\left(\mathrm{TCNX}^{2-}\right.$ $\left(\mathrm{Ru}^{\mathrm{II}}\right)_{4}$ signifies intramolecular electron reorganization back in the direction of the starting materials. The results described here indicate a dichotomy (Scheme 7) between those systems like $\left\{\left(\mu_{4}-\mathrm{TCNQ}\right)\left[\operatorname{Re}(\mathrm{bpy})(\mathrm{CO})_{3}\right]_{4}\right\}^{4+}{ }^{10}$ and $\left\{\left(\mu_{4^{-}}\right.\right.$ TCNX) $\left.\left[\mathrm{Fe}\left(\mathrm{C}_{5} \mathrm{H}_{5}\right)(\mathrm{CO})_{2}\right]_{4}\right\}^{4+15}$ which show no signs of metal-to-TCNX electron transfer and paramagnetic compounds such as $\left\{\left(\mu_{4}-\mathrm{TCNX}\right)\left[\mathrm{Ru}\left(\mathrm{NH}_{3}\right)_{5}\right]_{4}\right\}^{8+}$ ${ }^{11},\left\{\left(\mu_{4}-\mathrm{TCNX}\right)\left[\mathrm{Mn}\left(\mathrm{C}_{5} \mathrm{R}_{5}\right)(\mathrm{CO})_{2}\right]_{4}\right\} \quad 9 \quad\left\{\left(\mu_{4}-\mathrm{TCNX}\right)\left[\mathrm{Os}(\mathrm{H})(\mathrm{Cl})\left(\mathrm{PR}_{3}\right)_{2}(\mathrm{CO})\right]_{4}\right.$ \}$^{4+12}$ or $\left\{\left(\mu_{4}-\mathrm{TCNQ}\right)\left[\mathrm{Fe}\left(\mathrm{C}_{5} \mathrm{H}_{5}\right)(\mathrm{dppe})\right]_{4}\right\}^{4+14}$ which exhibit spectroscopic and electrochemical signs of significantly reduced bridging TCNX ligands - a requirement for the formation of molecular magnets ${ }^{2,3}$. Both cases are extreme formulations of the general description in Scheme 4, illustrating absent or complete metal-to-ligand electron transfer. It may be speculated that intermediate situations $(\delta=1)$ with genuine radical anion radical bridges can lead to the pronounced magnetism which has been observed for the roomtemperature magnet $\left[\mathrm{V}(\mathrm{TCNE})_{2}\right] \cdot \mathrm{x}$ solvent ${ }^{2 \mathrm{a}, 18}$ and related species ${ }^{25}$.

\section{Scheme 7}

\begin{tabular}{|l|l|}
\hline $\begin{array}{l}\text { no intramolecular electron } \\
\text { transfer }\end{array}$ & intramolecular electron transfer \\
\hline$\left\{\left(\mu_{4}-\mathrm{TCNQ}^{0}\right)\left[\mathrm{Re}^{\mathrm{I}}(\mathrm{CO})_{3}(\mathrm{bpy})\right]_{4}\right\}^{4+}$ & $\left\{\left(\mu_{4}-\mathrm{TCNX}^{2}\right)\left[\mathrm{Ru}^{2.5}\left(\mathrm{NH}_{3}\right)_{5}\right]_{4}\right\}^{8+}$ \\
\hline$\left\{\left(\mu_{4}-\mathrm{TCNX}^{0}\right)\left[\mathrm{Fe}^{\mathrm{II}}(\mathrm{CO})_{2}\left(\mathrm{C}_{5} \mathrm{H}_{5}\right)\right]_{4}\right\}^{4+}$ & $\left\{\left(\mu_{4}-\mathrm{TCNX}^{2}\right)\left[\mathrm{Mn}^{1.5}(\mathrm{CO})_{2}\left(\mathrm{C}_{5} \mathrm{R}_{5}\right)\right]_{4}\right\}^{0}$ \\
\hline
\end{tabular}

\section{ACKNOWLEDGEMENTS}

This work has been supported by the Land Baden-Württemberg, the Fonds der Chemischen Industrie and the Deutsche Forschungsgemeinschaft.

\section{REFERENCES}

1. (a) W. Kaim, M. Moscherosch, Coord. Chem. Rev. 129, 157, (1994). (b) J. S. Miller, Angew. Chem. 118, 2570, (2006); Angew. Chem. Int. Ed. 45, 2508, (2006).

2. (a) J. M. Manriquez, G. T. Yee, R. S. McLean, A. J. Epstein, J. S. Miller,. Science 252, 1415, (1991). (b) E. B. Vickers, T. D. Selby, M. S. Thorum, M. L. Taliaferro, J. S. Miller, Inorg. Chem. 43, 6414, (2004). (c) M. L. Taliaferro, M. S. Thorum, J. S. Miller, Angew. Chem. 118, 5452, (2006); Angew. Chem. Int. Ed. 45, 5326, (2006).

3. (a) H. Miyasaki, T. Izawa, N. Takahashi, M. Yamashita, K. R. Dunbar, J. Am. Chem. Soc. 128, 11358, (2006). (b) G. Wang, H. Zhu, J. Fan, C. Siebodnick, G. T. Yee, Inorg. Chem. 45, 1406, (2006). (c) R. Jain, K. Kabir, J. B. Gilroy, K. A. R. Mitchell, K.-C. Wong, R. G. Hicks, Nature 445, 291, (2007).

4. (a) R. S. Potember, T. O. Poehler, D. O. Cowan, Appl. Phys. Lett. 405, (1979). (b) K. Xiao, J. Tao, Z. Pan, A. A. Puretzky, I. N. Ivanov, S. J. Pennycook, D. B. Geohegan, Angew. Chem. 119, 22704, (2007); Angew. Chem. Int. Ed. 46, 2650, (2007). (c) A. Nafady, A. M. Bond, A. Bilyk, A. R. Harris, A. I. Bhatt, A. P. O'Mullane, R. De Marco, J. Am. Chem. Soc. 129, 2369, (2007).

5. S. B. Braun-Sand, O. Wiest, J. Phys. Chem. A107, 285, (2003).

6. H. Hartmann, B. Sarkar, W. Kaim, J. Fiedler, J. Organomet. Chem. 687, 100, (2003).

7. F. Baumann, M. Heilmann, W. Matheis, A. Schulz, W. Kaim, J. Jordanov, Inorg. Chim. Acta 251, 239, (1996).

8 B. Olbrich-Deussner, W. Kaim, R. Gross-Lannert, Inorg. Chem. 28, 3113 , (1989).

9. (a) R. Gross, W. Kaim, Angew. Chem. 99, 257, (1987); Angew. Chem. Int. Ed. Engl. 26, 251, (1987). (b) R. Gross-Lannert, W. Kaim, B. OlbrichDeussner, Inorg. Chem. 29, 5046, (1990).

10. (a) H. Hartmann, W. Kaim, I. Hartenbach, T. Schleid, M. Wanner, J. Fiedler, Angew. Chem. 113, 2927, (2001); Angew. Chem. Int. Ed. 40, 2842, (2001). (b) H. Hartmann, W. Kaim, M. Wanner, A. Klein, S. Frantz, C. Duboc-Toia, J. Fiedler, S. Zalis, Inorg. Chem. 42, 7018, (2003). (c) M. Leirer, G. Knör, A. Vogler, Inorg. Chem. Commun. 2, 110, (1999).

11. (a) M. Moscherosch, E. Waldhör, H. Binder, W. Kaim, J. Fiedler, Inorg. Chem. 34, 4326, (1995). (b) E. Waldhör, W. Kaim, M. Lawson, J. Jordanov, Inorg. Chem. 36, 3248, (1997). (c) S. Záliš, W. Kaim, B. Sarkar, C. Duboc, unpublished.

12. F. Baumann, W. Kaim, J. A. Olabe, A. Parisse, J. Jordanov, J. Chem. Soc., Dalton Trans., 4455, (1997).

13. S. Berger, H. Hartmann, M. Wanner, J. Fiedler, W. Kaim, Inorg. Chim. Acta 314, 22, (2001).

14. C. Diaz, A. Arancibia, Polyhedron 19, 137, (2000).

15. (a) A. N. Maity, B. Schwederski, W. Kaim, Inorg. Chem. Commun. 8, 600, (2005). (b) A. N. Maity, B. Schwederski, B. Sarkar, S. Záliš, J. Fiedler, S. Kar, G. K. Lahiri, C. Duboc, M. Grunert, P. Gütlich, W. Kaim, Inorg. Chem. 46, 7312 (2007).

16. F. A. Cotton, Y. Kim, J. Am. Chem. Soc. 115, 8511, (1993).

17. (a) L Shields, J. Chem. Soc. Farady Trans. 2 81, 1, (1985). (b) S. A. O'Kane, R. Clérac, H. Zhao, X. Ouyang, J. R. Galán-Mascarós, R. Heintz, K. R. Dunbar, J. Solid State Chem. 152, 159, (2000). (c) C. Campana, K. R. Dunbar, X. Ouyang, Chem. Commun., 2427, (1996). (d) H. Miyasaka, 
C. S. Campos-Fernández, R. Clérac, K. R. Dunbar, Angew. Chem. 112, 3989, (2000); Angew. Chem. Int. Ed. Engl. 39, 3831, (2000). (e) R. A. Heintz; H. Zhao, X. Ouyang, G. Grandinetti, J. Cowen, K. R. Dunbar, Inorg. Chem. 38, 144, (1999).

18. G. Wang, H. Zhu, C. Slebodnick, G. T. Yee, Inorg. Chem. 45, 1406, (2006).

19. K. I. Pokhodnya, A. J. Epstein, J. S. Miller, Adv. Mater. 12, 410, (2000).

20. J. S. Miller, A. J. Epstein, Angew. Chem. 106, 399, (1994); Angew. Chem. Int. Ed. Engl. 33, 385, (1994).

21. P. Chaudhuri, C. N. Verani, E. Bill, E. Bothe, T. Weyhermüller, K. Wieghardt, J. Am. Chem. Soc. 123, 2213, (2001).
22. C. Creutz, Prog. Inorg. Chem. 30, 1, (1983).

23. H. Krentzien, H. Taube, Inorg. Chem. 21, 4001, (1982)

24. W. Kaim, G. K. Lahiri, Angew. Chem. 119, 1808, (2007); Angew. Chem. Int. Ed. 46, 1778, (2007).

25. (a) K. I. Pokhodnya, M. Bonner, J.-H. Her, P. W. Stephens, J. S. Miller, J. Am. Chem. Soc. 128, 15592, (2006). (b) J.-H. Her, P. W. Stephens, K. I. Pokhodnya, M. Bonner, J. S. Miller, Angew. Chem. 119, 1543, (2007); Angew. Chem. Int. Ed. 46, 1521, (2007). 